\title{
Efficient resource allocation for passive optical fronthaul-based coordinated multipoint transmission
}

\author{
Gang Wang ${ }^{1,2}$, Rentao $\mathrm{Gu}^{1,2^{*}}$ (D) Hui Li $\mathrm{i}^{1,2}$ and Yuefeng $\mathrm{Ji}^{3,2^{*}}$
}

\begin{abstract}
The centralized processing in cloud radio access network enables cooperation between baseband processing units (BBUs) like inter-cell interference (ICI) cancellation on the basis of coordinated multipoint (CoMP). Large amounts of the sharing data will be transmitted through fronthaul transport network. In the paper, both integer non-linear programming (INLP) optimization model and adaptive genetic algorithm (GA) are explored to release the capacity pressure of the fronthaul transport network when CoMP is introduced. We also consider the resource allocation problem of the passive optical fronthaul network. The proposed algorithm tries to reduce the downlink bandwidth and improve the optical resource allocation efficiency of the optical fronthaul with minimal influence on the fronthaul topology. During the simulations, three critical factors are considered: (1) the number of cell edge users, (2) the average traffic demand of cell edge users, (3) the size of cell cluster used to enable the CoMP. The simulation results show that the most efficient bandwidth saving and optical resource allocation can be achieved with INLP, while the proposed adaptive GA nearly has the same performance with low computational complexity and fast convergence, which is more applicable for the large-scale fronthaul network. Furthermore, the load difference of the fronthaul transport network can be further reduced.
\end{abstract}

Keywords: Network optimization, INLP, Coordinated multipoint, Adaptive genetic algorithm, TWDM-PON-enabled fronthaul

\section{Introduction}

Network densification using small cells is emerging as a critical technology to enhance the resource management of next-generation wireless network $[1,2]$. However, the received signal quality of cell edge users can be sharply degraded by the transmission of neighboring cells. Thus, the signal-to-noise ratio is badly influenced (particularly near the cell edge), as well as the downlink capacity of the mobile network. Inter-cell interference (ICI) has been a bottleneck to improve the mobile network capacity and the quality of service (QoS) of the mobile users that located at cell edge [3]. To face this challenge, coordinated multipoint (CoMP) was proposed $[4,5]$. Multiple base stations (BSs)

\footnotetext{
* Correspondence: rentaogu@bupt.edu.cn; jyf@bupt.edu.cn

'Beijing Key Laboratory of Network System Architecture and Convergence, School of Information and Communication Engineering, Beijing University of Posts and Telecommunications, Beijing 100876, China

${ }^{3}$ State Key Laboratory of Information Photonics and Optical Communications, School of Information and Communication Engineering, Beijing University of Posts and Telecommunications, Beijing 100876, China

Full list of author information is available at the end of the article
}

are connected and exchange information for cooperation via backhaul links to reduce ICI. And prediction algorithms can be used to estimate the movement of mobile equipment $[6,7]$. Techniques enabling coordinated transmission are explored to migrate the inter-cell interference and increase the system capacity [8-16]. Spectral efficiency (SE)oriented CoMP techniques have been investigated [8-10]. In $[8,9]$, CoMP precoders have been explored to improve the spectral efficiency and network capacity. And both spectral efficiency and fairness were considered in CoMP systems [10]. Besides, energy efficiency (EE)-oriented CoMP techniques have also been studied [11-14]. In CoMP-enabled mobile network, the authors investigated the downlink transmit power optimization problem with QoS constraint and limited cell coordination (max-min EE for CoMP systems) [11]. Considering the individual data rate requirement and transmit power of each $\mathrm{BS}$, energyefficient CoMP precoding was proposed [12]. And EEoriented resource allocation algorithm was also proposed in CoMP-enabled heterogeneous network, considering of 
the backhaul power consumption [13]. Semi-smart antenna-based coordinated multipoint technique has been studied to reduce the transmit power of orthogonal frequency division multiplexing (OFDMA) networks [14]. Furthermore, the methods to acquire channel state information were discussed in [5]. Limited feedback CoMP system was reviewed in [15]. Moreover, in [16], the authors took the non-ideal backhaul into consideration, and the spectrum allocation scheme was proposed in heterogeneous network for coordinated multipoint transmission. And cooperation between base stations in the downlink of heterogeneous network has been studied [17]. In [18], CoMP downlink transmission design for cloud radio access network (C-RAN) was studied.

C-RAN is emerging as a potential architecture for the next-generation wireless network [19]. In C-RAN, baseband units (BBUs) are migrated and centralized into a BBU central server retaining only distributed remote radio units (RRUs) at remote cells [20]. The fiber technique-based network used to forwarding signals between BBUs and RRUs is called fronthaul [21]. This novel architecture opens up opportunities for a better management of resource of the mobile network.

CoMP can benefit from the centralized processing in CRAN. However, large amounts of sharing data need to be transmitted in the fronthaul transport network when CoMP is introduced in C-RAN. Considering the limited bandwidth and optical resource of the fronthaul, to release the capacity pressure and improve the optical resource efficiency of the fronthaul is significantly important when CoMP technique is introduced in next-generation radio access network. However, as far as we know, little attention has been focused on the influence on fronthaul when CoMP technique is introduced in C-RAN. In previous works, recent advances like key technologies and system architectures in fronthaul-constrained C-RAN have been discussed in [22]. Advanced techniques were explored to enhance the utilization efficiency and transfer capability of the optical fronthaul [23-29]. Digital signal processing (DSP)-based channel aggregating technique was researched [23]. To ensure a reasonable fronthaul transmission rate, subcarrier multiplexing technique was investigated [24]. In [25], microwave-photonics techniques were introduced for integrated optical-wireless access network. Besides, topology-reconfigurable fronthaul transport network has been proposed [26]. CoMP and device-todevice (D2D) connectivity can benefit from this architecture and network measurement schemes [30] in the 5G mobile networking era. And different models of optical fronthaul for C-RAN were discussed [27]. Furthermore, to simplify the RRU, fully passive RRU and self-tuning colorless optical network unit (ONU) transmitter was proposed and demonstrated for short-range wireless network [28]. Data and energy are jointly transmitted through optical fronthaul.
Moreover, in C-RAN with non-ideal fronthaul network, delay-sensitive services can benefit from the efficient strategy proposed in [29]. And multicore fiber media (MCF) has been investigated for the future optical fronthaul [31].

However, little work has been done to solve CoMPoriented resource allocation problem in the fronthaul transport. In the paper, we try to release the capacity pressure and improve the optical resource allocation efficiency of the fronthaul with minimal influence on the fronthaul topology. We present two CoMP-oriented resource allocation schemes for the fronthaul transport network. Both integer non-linear programming (INLP) model and adaptive genetic algorithm (GA) are explored.

In this paper, we formulate the INLP model in Section 2, and Section 3 discusses the adaptive GA. Meanwhile, in Section 4, the performance of numerical simulations are described. Finally, we summarize the paper in Section 5.

\section{INLP model formulation for CoMP-oriented fronthaul}

In subsequent subsections, we present the CoMP-oriented C-RAN architecture and develop the INLP model to release the capacity pressure and improve the optical resource allocation efficiency of the time and wavelength division multiplexing (TWDM) passive optical network (PON)-enabled fronthaul transport network.

\subsection{CoMP-oriented C-RAN architecture}

Figure 1 illustrates the CoMP-oriented C-RAN architecture. Recently, large-scale deployment of PONs significantly releases the capacity pressure of the access network $[32,33]$. TWDM-PON is emerging as a potential candidate to transfer data between centralized BBUs and distributed RRUs with strong ability of transmission [34]. As shown in Fig. 1, optical line terminal is deployed at BBU pool, and ONUs with tunable lasers are placed with cost and power efficient RRUs. Virtualization technology has been widely investigated $[35,36]$. In TWDM-PON-enabled fronthaul, a virtual PON is formed when a group of ONUs transfer data using the same wavelength. As shown in [27], based on the software defined network (SDN) technique, different transport abstractions can be achieved in the centralized controller, which results in better performance of the C-RAN. Besides, we also take common public radio interface (CPRI) compression techniques [37-39] into consideration, since the typical CPRI physical link rate is fixed [19]. Compared to the typical CPRI, compression techniquebased bitrate-variable CPRI is a potential efficient radio interface to face the challenge of overwhelming data stream in the $5 \mathrm{G}$.

\subsection{INLP model formulation}

In [8-16], different challenges of CoMP technique have been investigated, such as SE/EE-oriented precoder, the 


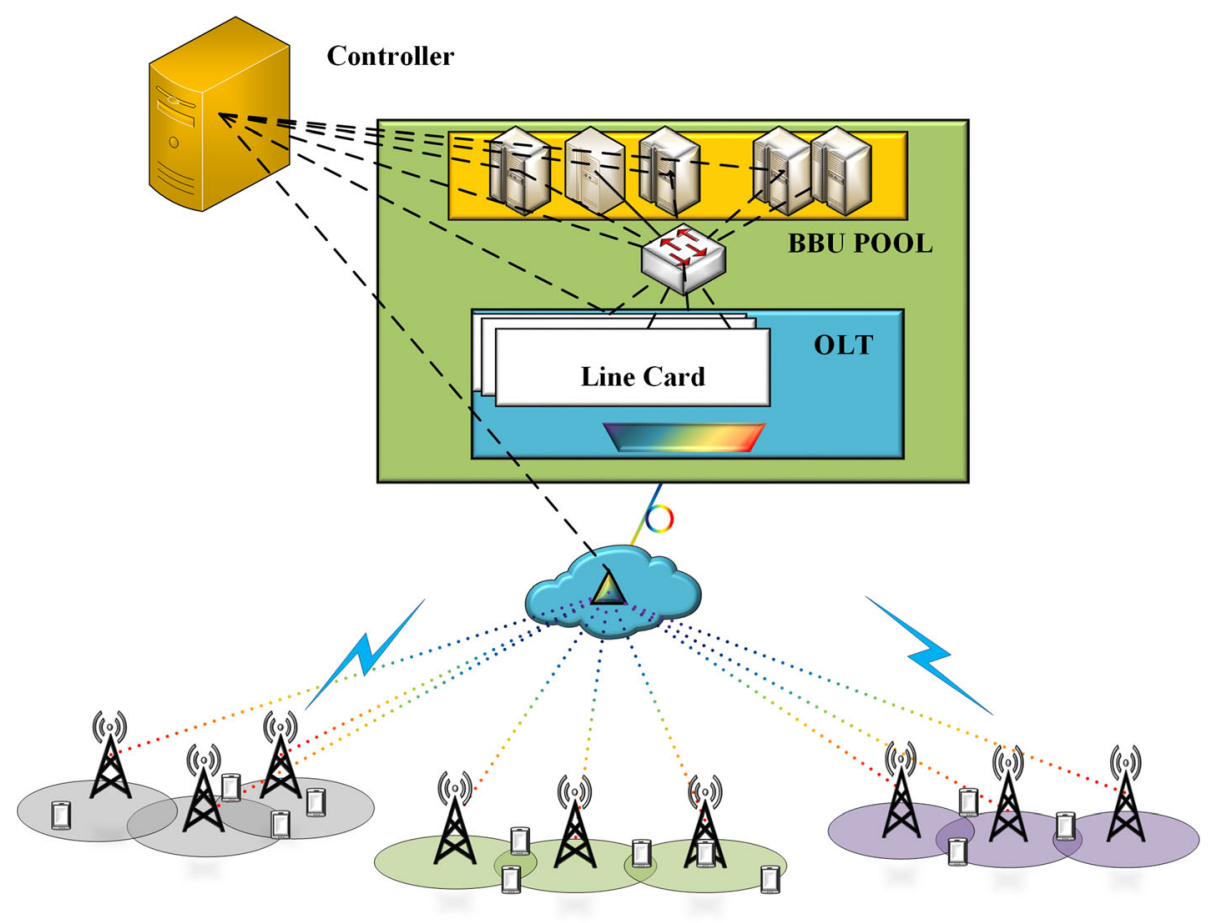

Fig. 1 CoMP-oriented C-RAN architecture

influence of the limited feedback. However, little attention has been focused on the influence on fronthaul when CoMP technique is introduced in C-RAN. Considering that large sharing data needs to be transmitted in the fronthaul transport network when CoMP technique is introduced, we focus on releasing the capacity pressure and improving the optical resource allocation efficiency of the fronthaul with minimal influence on the fronthaul topology. The proposed INLP model considers CoMP technique and broadcast characteristic of the TWDM-PON. The transmission of sharing data can benefit greatly from the broadcast characteristic of the TWDM-PON.

Notations:

- W: The set of optical wavelength resources used in the TWDM-PON-enabled fronthaul transport network.

- C: The set of cells served by the TWDM-PONenabled C-RAN.

- O: The set of distributed ONUs at cell sites colocated with simplified RRUs.

- T: A series of discrete time slots.

- I: The set of mobile terminals located at the small cells.

- Ie: The set of cell edge mobile terminals, Ie $\subset \mathrm{I}$.

- $\mathrm{C} i$ : The small-cell cluster enabling CoMP for cell edge mobile terminal $i, i \in \mathrm{I} e, \mathrm{C} i \in \mathrm{C}$.

- $C v$ : The maximum bandwidth of a single-wavelength. In current TWDM-PON system, $C v=10 \mathrm{~Gb} / \mathrm{s}$.
- $\quad v c$ : CPRI fixed link rate [19].

- ni: The size of small cell cluster enabling CoMP for cell edge mobile terminal $i, i \in \mathrm{I} e$.

- $b i$ : The bandwidth requirement of mobile terminal $i, i \in \mathrm{I}$.

- $\beta_{c, o}^{t}$ : Load fluctuation-based compression ratio of typical CPRI for cell $c$ served by ONU $o$ at time $t, c \in \mathrm{C}, o \in \mathrm{O}$.

- $\mathrm{Q}_{c, o}^{t}=\{i \mid i \in \mathrm{I} i$ is in cell $c$ at time $t\}$ : The set of mobile terminals that is located in the small cell $c$ is served by the corresponding ONU $o$.

- $\mathrm{R} w, o j=\{w \mid w \in \mathrm{W} j, \mathrm{~W} j \subseteq \mathrm{W}\}$ : The wavelength tuning range of ONU oj, oj $\in \mathrm{O}$.

- $\Omega_{w, o}^{t}$ : The topology of current TWDM-PONenabled fronthaul at time $t$.

Variable:

$\lambda_{w}^{t}=\left\{\begin{array}{cc}1, & \text { if wavelength } w \in \mathbf{W} \text { is used for data transmission } \\ 0, & \text { at time } t\end{array}\right.$

$\boldsymbol{\Omega}_{w, o}{ }^{t}=\left\{\begin{array}{lc}1, & \text { if wavelength } w \in \mathbf{W} \text { is used to establish a } \\ & \text { lightpath for ONU } o \in \mathbf{O} \text { at time } t \\ 0, & \text { otherwise }\end{array}\right.$

$\sigma_{i}^{t}$ : The number of wavelength used for cell cluster $\mathbf{C} i$ enabling CoMP at time $t$. 
Objective:

Maximize the fronthaul transport network bandwidth allocation efficiency $\eta$ :

$$
\eta=\frac{\sum_{c \in \mathbf{C}} \sum_{i \in \operatorname{Ien} \cap \mathbf{Q}_{c, o}} n i b i-\sum_{c \in \mathbf{C}} \sum_{i \in \operatorname{Ien} \mathbf{Q}_{c, o}} \sigma_{i}^{t} b i}{\sum_{c \in \mathbf{C}} \sum_{i \in \operatorname{Ien} \mathbf{Q}_{c, o}} n i b i+\sum_{c \in \mathbf{C}} \sum_{i \in(\mathbf{I}-\mathbf{I} e) \cap \mathbf{Q}_{c, o}} b i}
$$

In addition, improving the optical resource allocation efficiency of the fronthaul plays a critical role for CoMPoriented optimization. Three sub-objectives are also considered during the optimization:

$$
\min \zeta=(\zeta 1, \zeta 2, \zeta 3)
$$

where:

$$
\begin{aligned}
\zeta 1 & =\sum_{w \in \mathbf{W}} \lambda_{w}^{t} \\
\zeta 2 & =\frac{\sum_{w \in \mathbf{W}}\left(\lambda_{w}^{t}\left(\sum_{o \in \mathbf{O}}\left(\Omega_{w, o}^{t} \cdot v c \cdot \beta_{c, o}^{t}\right)-\sum_{o \in \mathbf{O}} v c \cdot \beta_{c, o}^{t} / \sum_{w \in \mathbf{W}} \lambda_{w}^{t}\right)^{2}\right)}{\sum_{w \in \mathbf{W}} \lambda_{w}^{t}} \\
\zeta 3 & =\left(\sum_{w \in \mathbf{W}} \sum_{o \in \mathbf{O}}\left(\left(\Omega_{w, o}^{t}-\Omega_{w, o}^{t-1}\right)^{2} \cdot \sum_{i \in \mathbf{Q}_{c, o}^{t}} b i\right)\right) / 2
\end{aligned}
$$

Subject to

$$
\begin{aligned}
& \lambda_{w}^{t} \in\{0,1\}, \Omega_{w, o}^{t} \in\{0,1\} \quad \forall w \in \mathbf{W}, \forall o \in \mathbf{O} \\
& \sum_{w \in \mathbf{W}} \Omega_{w, o}^{t}=1 \quad \forall o \in \mathbf{O} \\
& \sum_{w \in \mathbf{W}} \lambda_{w}^{t} \leq|\mathbf{W}| \\
& \sum_{o \in \mathbf{O}} \Omega_{w, o}^{t} \cdot v c \cdot \beta_{c, o}^{t} \leq C v \quad \forall w \in \mathbf{W} \\
& w=\left\{w \mid \Omega_{w, o}^{t}=1, w \in \mathbf{W}\right\} \in \mathbf{R} w, o \quad \forall o \in \mathbf{O}
\end{aligned}
$$

The three sub-objectives of the INLP are as follows: (1) minimize the used optical resource, (2) balance out the traffic load served by the activated wavelengths, and (3) minimize the migrated load due to fronthaul topology adjustment. Equation (6) indicates the reasonable integer value of variable $\lambda_{w}^{t}$ and $\Omega_{w, o}^{t}, \forall w \in \mathbf{W}, \forall o \in \mathbf{O}$. Equation (7) states that each ONU co-located with the RRU at small cell can only be allocated one wavelength at time $t$. Equation (8) limits the maximum feasible wavelengths of fronthaul at time $t$. Equation (9) limits the maximum load served by each single-wavelength
$\mathrm{W}(\forall w \in \mathbf{W})$ at time $t$. Equation (10) ensures that the wavelength assigned to ONU $o(o \in \mathbf{O})$ is within the tuning range at time $t$.

\section{Adaptive genetic algorithm for CoMP-oriented resource allocation}

The complexity of INLP is exponentially increasing with the growing of network scale. To reduce the time complexity, an adaptive GA is proposed to solve the CoMP-oriented capacity and resource allocation problems of the fronthaul network. We will introduce the modified genetic encoding scheme, corresponding fitness function, and adaptive genetic operations for the proposed GA as follows.

\subsection{Genetic encoding and the fitness function}

GA is an efficient search heuristic method on the basis of principles of natural evolution in the real world [40]. A reasonable chromosome (or an individual) is encoded as a group of genes. For the CoMPoriented resource allocation, we encode each gene as $\{\xi(o j, w j), o j \in \mathbf{O}, w j \in \mathbf{R} w, o j\}$, where $\xi(o j, w j)$ indicates that wavelength $w j$ is allocated to ONU oj. For each distributed ONU oj, a wavelength $w j$ is randomly selected for its data transmission according to the traffic in the corresponding small cell $c j$. The lightpath is built up for data transmission between ONU oj and OLT. We apply this process for all ONUs to obtain an individual $I$. We can form a different individual by choosing different optical resource for some of genes. We randomly repeat $P$ times to generate more individuals and form the population $I$ by grouping different individuals together. In order to release the capacity pressure of the fronthaul when CoMP technique is introduced, we need to enhance the bandwidth efficiency of the optical fronthaul based on the broadcast characteristic of the TWDM-PON. In addition, improving the optical resource allocation efficiency of the fronthaul is also playing a significant role. Furthermore, we also need to pay attention to load balancing of the fronthaul and the influenced traffic load during ONU migration. Finally, each of the individual's fitness is assigned as $(\rho 1, \rho 2, \rho 3, \rho 4)(\eta$, $\zeta)^{\prime}$, where $\rho 1, \rho 2, \rho 3$, and $\rho 4$ are the weights allocated to the optimization objectives described in Section 2.2, respectively. Better individuals survive and reproduce themselves more often than the worse ones. In each iteration, we update the fittest individual on the basis of each individual's fitness. The GA can obtain a good result when it converges [41].

\subsection{Adaptive genetic operations}

Algorithm 1 illustrates the procedure of the proposed adaptive GA. The initial population I of constant size 
$P$ is generated randomly based on the gene generation principle mentioned above. Then the population I goes into the following adaptive genetic operations. The tournament selection [42] is adopted for the selection operation. We randomly select $s$ individuals from population I and implement tournament selection by holding a tournament among $s$ competitors, where $s$ is the tournament size. When all tournaments are finished, we select the winner of each competing group for crossover, on the basis of each individual's fitness among competitors.

In crossover phase, we implement multipoint gene level crossover to generate the offspring. We randomly select two individuals paired as parent for crossover. In each crossover operation, on the basis of crossover rate $p c,|\mathbf{O}| \cdot p c$ genes are randomly picked out from the parent and swapped at random positions of the individuals. Then $P$ individuals are selected based on their fitness to go into mutation phase. During the evolution, the population size is constant.

In the mutation phase, on the basis of mutation rate $p m$, $|\mathbf{O}| \cdot p m$ genes of the individuals will be randomly modified to generate new genes. And we modify a gene $\xi(o j, w j)$ by replacing its optical resource $w j$ with another feasible one. Based on the fitness of each individual, $P$ individuals are selected to form a new population. During crossover and mutation phases, in order to limit the maximum traffic load served by each single-wavelength and ensure that the ONU tuning range is legal, a penalty function is used during each genetic operation.

In the evolution phase, $p c$ and $p m$ vary with the fitness value of the initial population of each iteration. We define $F j$ as the fitness of individual $I j$. We have $F \max =$ $\max j(F j), \quad j \in \mathbf{I}, F$ mean $=\left(\sum_{j} F j\right) / P$, and $F^{\prime}=\max (F j 1$, $F j 2$ ). Then $p c$ and $p m$ are obtained by Eqn. (11) and Eqn. (12) [43], where $\beta c$ and $\beta m$ are fixed parameters.

$$
\begin{aligned}
& p c=\left\{\begin{array}{cc}
\frac{F \max -F^{\prime}}{F \max -F \operatorname{mean}}, & \begin{array}{c}
F^{\prime} \leq F \text { mean } \\
\beta c,
\end{array} \\
\text { otherwise }
\end{array}\right. \\
& p m=\left\{\begin{array}{cc}
\frac{F \max -F j}{F \max -F \operatorname{mean}}, & F p \leq F \text { mean } \\
\beta m, & \text { otherwise }
\end{array},\right.
\end{aligned}
$$

We define GA's degree of diversity as Eqn. (13) [44]. $d(j 1, j 2)$ indicates the differences between two individuals $I j 1$ and $I j 2$. The GA stopped when its convergence

\begin{tabular}{|c|c|}
\hline $\begin{array}{l}\text { Algorithm } 1 \text { CoMP Oriented Genetic Algorithm for Passive } \\
\text { Optical Fronthaul }\end{array}$ & $\begin{array}{l}\text { Algorithm } 1 \text { CoMP Oriented Genetic Algorithm for Passive } \\
\text { Optical Fronthaul }\end{array}$ \\
\hline $\begin{array}{l}\text { 1: } \mathbf{I}=\text { Null set; } \\
\{\text { Stage } 1: \text { Randomly Initialize the First Generation } \\
\quad \text { Population } \mathbf{I} \text { of Constant Size } P\} \\
\text { 2: while }|\mathbf{I}| \text { is less than } P \text { do } \\
\text { 3: } \quad I=\text { Null set; } \\
\quad\{\text { Generate an Individual } I\} \\
\text { 4: for each distributed ONU } o_{j} \in \mathbf{O} \text { do } \\
\text { 5: } \quad \text { randomly choose a reasonable wavelength } w_{j} ; \\
\text { 6: } \quad \text { generate a gene }\left\{\xi\left(o_{j}, w_{j}\right), o_{j} \in \mathbf{O}, w_{j} \in \mathbf{R}_{w, o_{j}}\right\} \\
\text { 7: } \quad \text { put gene }\left\{\xi\left(o_{j}, w_{j}\right), o_{j} \in \mathbf{O}, w_{j} \in \mathbf{R}_{w, o_{j}}\right\} \text { in } I ; \\
\text { 8: end for } \\
\text { 9: check the individual } I ; \\
\text { 10: if } I \text { is a reasonable individual then } \\
\text { 11: put } I \text { in } \mathbf{I} ; \\
\text { 12: end while } \\
\{\text { Stage } 2: \text { Adaptive Genetic Operations based Evolution }\} \\
\text { 13: Ibest }=\text { Null set; }\end{array}$ & $\begin{array}{l}\text { 14: while the proposed adaptive GA is not converged do } \\
\text { 15: calculate the fitness value }\left(\rho 1, \rho^{2}, \rho^{3}, \rho^{4}\right)(\eta, \zeta)^{\prime} \\
\text { for each individual; } \\
\text { 16: while number of tournaments is less than } P \text { do } \\
\text { 17: randomly select } s \text { individuals from current } \\
\text { population; } \\
\text { 18: select the winner of the competing group; } \\
\text { 19: end while; } \\
\text { 20: select the winner of each competing group for } \\
\quad \text { crossover, on the basis of each individual's fitness } \\
\text { among competitors; } \\
\text { 21: choose } P \text { fittest individuals based on their fitness } \\
\text { to go into mutation phase; } \\
\text { 22: Ibest } \leftarrow \text { the fittest individual in } \mathbf{I} \text { on the basis of } \\
\text { fitness }\left(\rho_{1}, \rho_{2}, \rho_{3}, \rho_{4}\right)(\eta, \zeta)^{\prime} \text {; } \\
\text { 23: adjust } p_{c} \text { and } p_{m} ; \\
\text { 24: update the } D_{p} \text { for } \mathbf{I} \text {; } \\
\text { 25: end while }\end{array}$ \\
\hline
\end{tabular}
reaches a preset threshold [41]. 


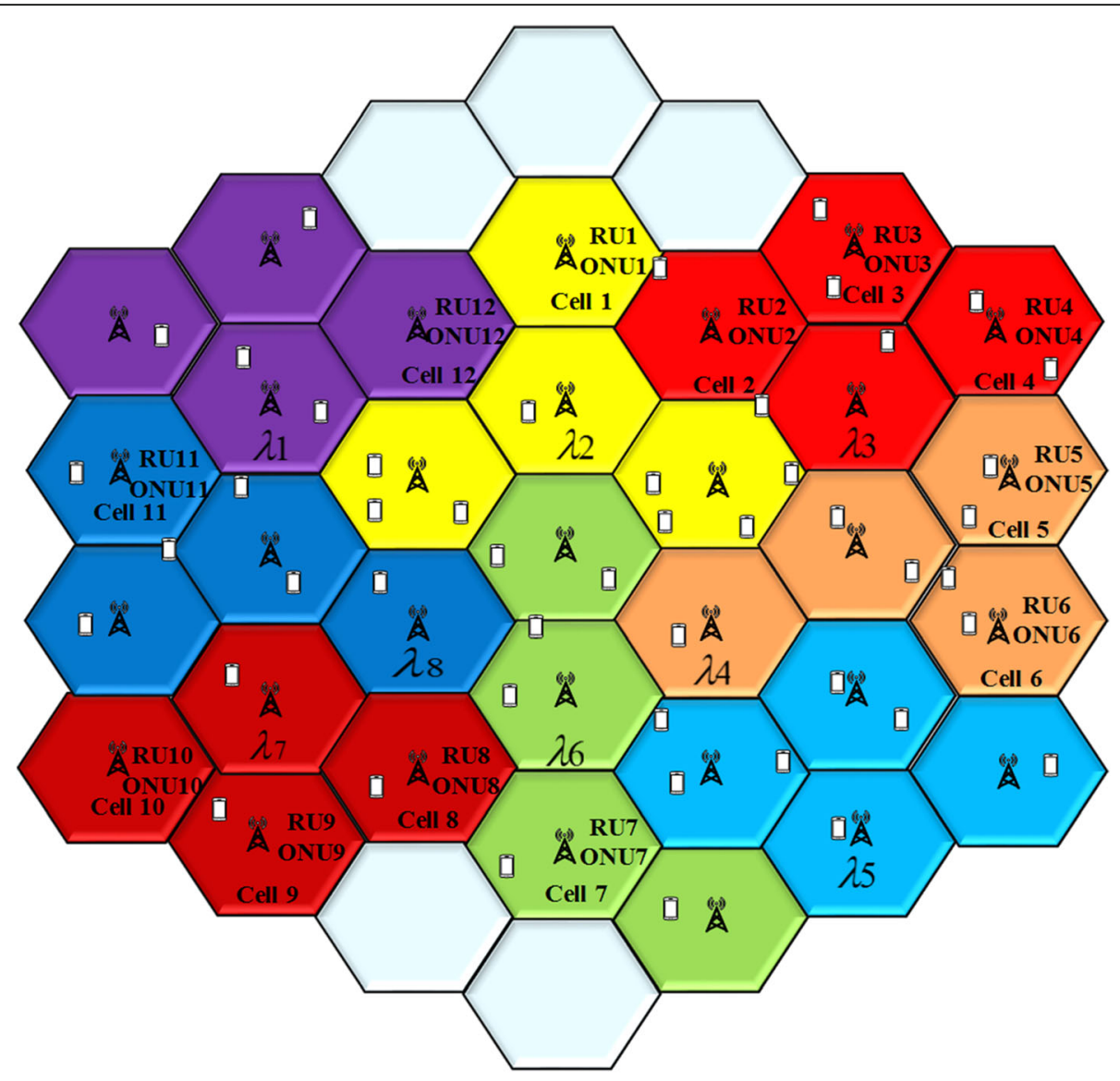

Fig. 2 The 32-cell topology for simulations

$$
D p=\frac{2}{P(P-1)} \sum_{j 1=1}^{P-1} \sum_{j 2=j 1+1}^{P} \frac{d(j 1, j 2)}{|I|}
$$

\section{Performance evaluations}

Numerical simulations are conducted based on the proposed INLP model and adaptive GA for CoMP-oriented optical resource optimization. In the simulations, a 32cell physical topology (shown in Fig. 2) is used, and the number of used wavelengths in the TWDM-PONenabled fronthaul is set as eight. We assume that each distributed RRU is assigned only one ONU with tunable lasers, and compression technique-based bitrate-variable CPRI is adopted in TWDM-PON-enabled fronthaul. Besides, we also assume that time is divided into discrete time periods. Table 1 illustrates the parameters used in our simulations.

In the simulations, different scenarios are considered: (1) typical C-RAN without CoMP-oriented optimization; (2) C-RAN with CoMP-oriented INLP optimization; 3) C-RAN with CoMP-oriented GA. We also consider the influence of the tuning range of the ONUs. With the traffic fluctuation, we try to release the capacity pressure and improve the resource allocation efficiency of the fronthaul when CoMP technique is introduced in CRAN. Besides, the migrated traffic due to topology change is also considered in our formulations.

Figure 3 represents the converging condition of the proposed adaptive GA with the $D p$ defined in Eqn. (13). It is obviously that the proposed GA converges when the number of iterations exceed 50, if the threshold of the

Table 1 Simulation parameters

\begin{tabular}{ll}
\hline$C V$, the maximum bandwidth of a single-wavelength & $10 \mathrm{Gbits} / \mathrm{s}$ \\
Bandwidth requirement of a mobile terminal & $0-$ \\
& $20 \mathrm{Mbits} / \mathrm{s}$ \\
$V C$, fixed physical link rate of typical CPRI [19] & $2.5 \mathrm{Gbits} / \mathrm{s}$ \\
$\beta_{c, o}^{t}$ load fluctuation-based compression ratio of typical & $0.2-1$ \\
$C P R I$ for cell $C$ served by ONU o & \\
The number of the distributed optical network units (ONUs) & 32 \\
$P$, the constant size of the initial population & 60 \\
The number of the mobile terminals & 100 \\
ni, the size of the cell cluster enabling the CoMP & 2 or 3 \\
Dp, preset convergence threshold of the proposed & 0.15 \\
adaptive GA & \\
\hline
\end{tabular}




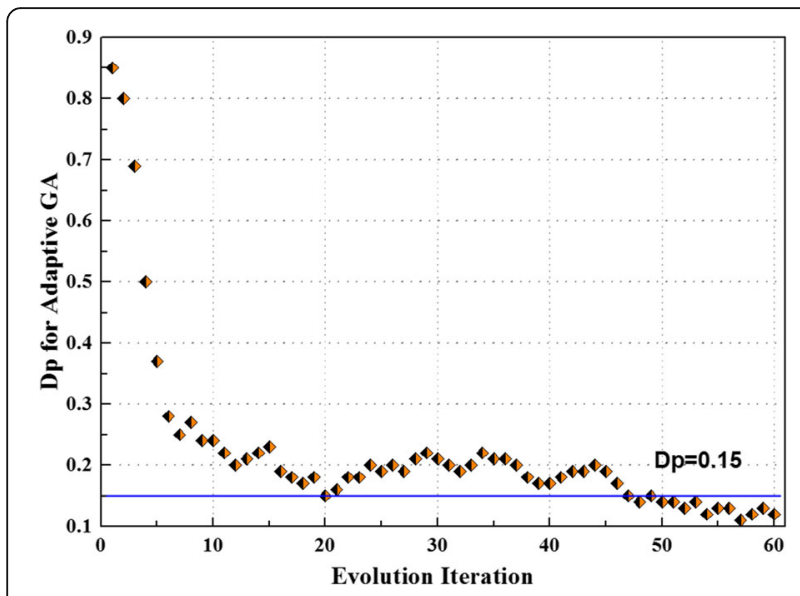

Fig. 3 Convergence performance of the adaptive GA

$D p$ is set at 0.15. Besides, compared to the INLP method, the computation time of the proposed GA is much lower within $1.68 \mathrm{~s}$.

Figure 4 illustrates the optical resource allocation performance comparison of the INLP to the proposed adaptive GA. The limited tuning range of each ONU is also considered. It is known that the traffic flow varies with the time in the fronthaul. Compared to the scenario without CoMP-oriented optimization, better optical resource allocation performance can be obtained with INLP. Specifically, in light traffic load time slot, nearly $70 \%$ wavelengths can be saved by using INLP. However, in typical C-RAN, wavelength resource allocation is fixed regardless of load variation. It is known that the complexity of INLP is exponentially increasing with the growing of network scale. As we can see, the proposed GA nearly has the same resource allocation performance with the INLP based on the load fluctuation. Compared to the INLP, the computation time of the adaptive GA is much lower within $1.68 \mathrm{~s}$. Besides, compared to the tuning limited INLP, better performance

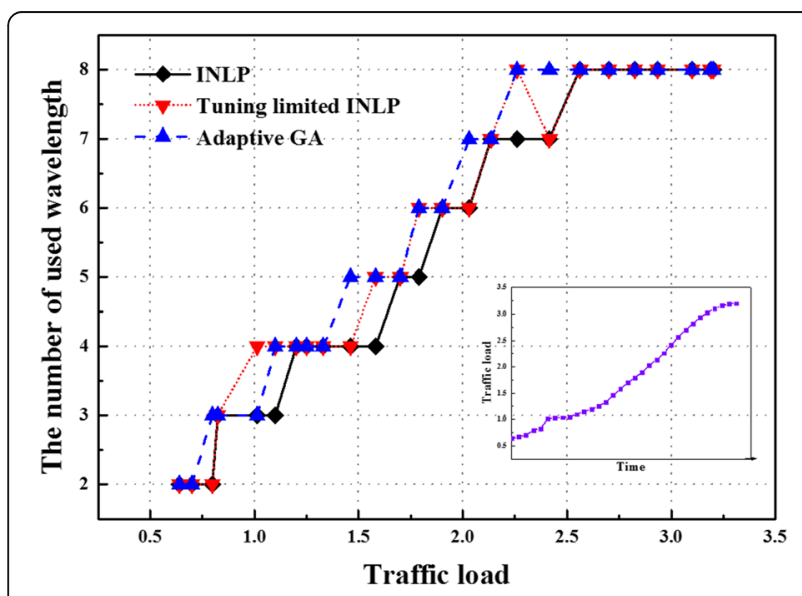

Fig. 4 Utilization of wavelength with different methods

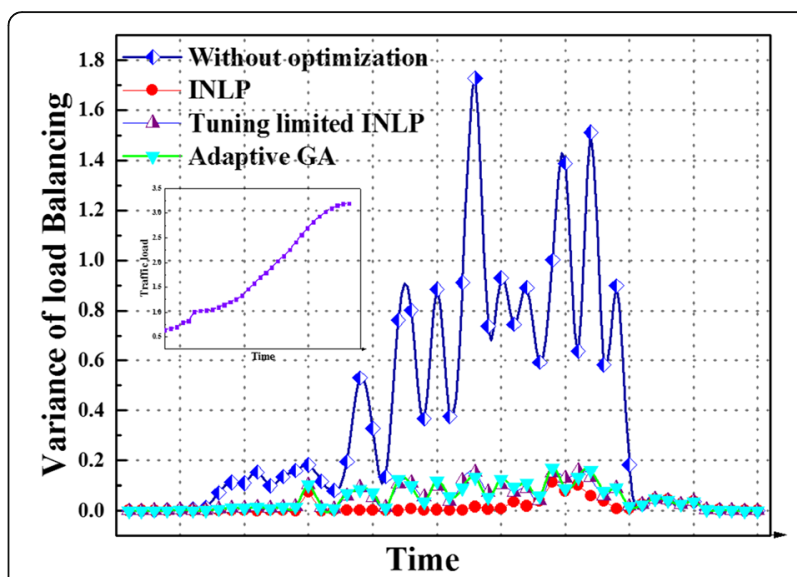

Fig. 5 Load balancing simulation results with different methods

can be obtained with full-spectrum tunable lasers, which is more expensive. Furthermore, as shown in Eqn. (4) and Eqn. (5), the load imbalance and the migrated traffic due to topology adjustment are also considered during the optimization. In traditional C-RAN, considering that the traffic served by each wavelength resource has a big difference, the load imbalance is clearly in the TWDM-PONenabled fronthaul. As shown in Fig. 5, the variance of the traffic load fluctuation is further reduced, while the migrated traffic load due to topology change is very light by using INLP and the proposed adaptive GA.

Figures 6, 7, 8, and 9 show the CoMP-oriented downlink bandwidth optimization simulation results with different methods. When the number of mobile users that located near the small cell edge is small, little sharing data is needed to be transmitted through fronthaul transport network. The performance of the proposed algorithms is not obvious. However, with the growth of mobile users that is near the cell edge, the sharing data is getting large. As shown in the Fig. 6, the most efficient bandwidth saving can be achieved by using INLP. Compared with INLP,

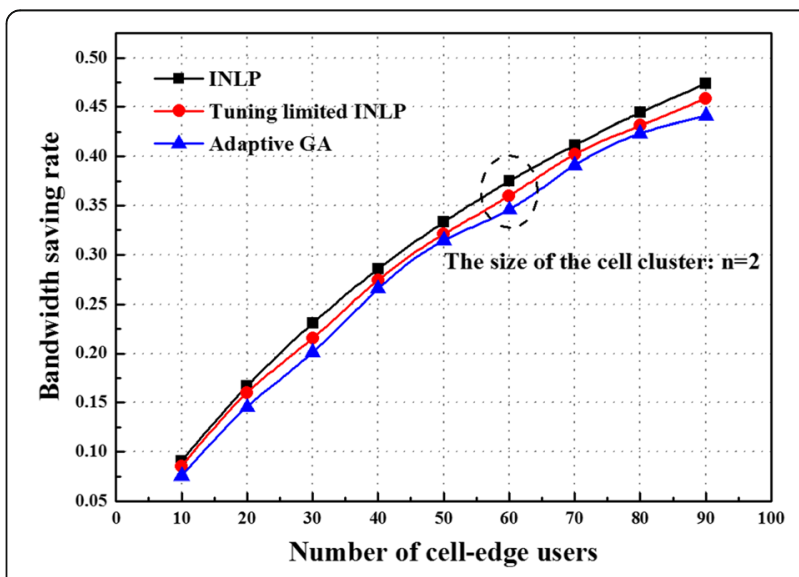

Fig. 6 Bandwidth saving rate considering the number of cell edge users with different methods $(n=2)$ 


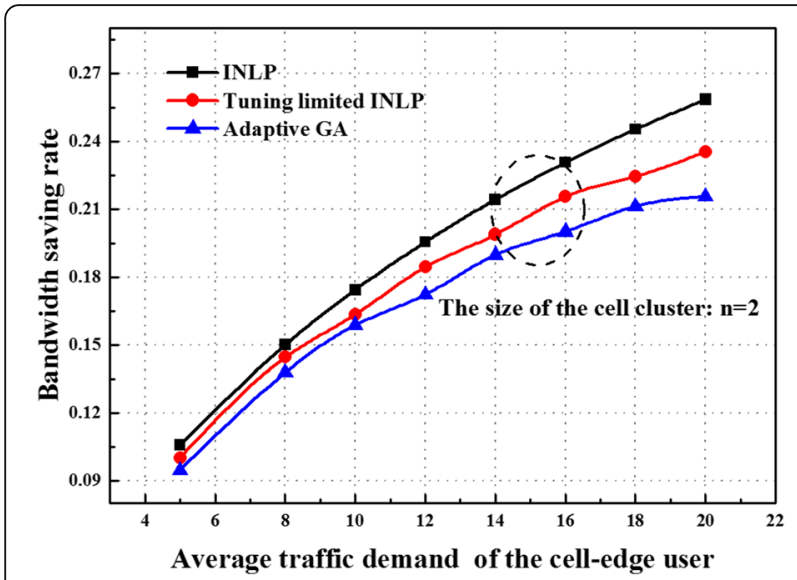

Fig. 7 Bandwidth saving rate considering the average traffic demand of cell edge users with different methods $(n=2)$

similar trends can be observed by using the proposed adaptive GA with lower computational complexity. The limited tuning range of the $\mathrm{ONU}$ is also considered. Better performance can be obtained with full-spectrum tunable lasers. Besides, Fig. 7 represents the influence of the average traffic demand on the optimization. When the bandwidth demand of the cell edge users increases, the total demand of the downlink bandwidth is increasing including the bandwidth allocated to the sharing data. By using the proposed method, the capacity pressure of the fronthaul is released. Furthermore, the size of the cell cluster used to enable the CoMP also plays a critical role in the optimization. As shown in Figs. 8 and 9, the larger the size of the cell cluster, the better performance can be achieved. Finally, the significant bandwidth saving is attributed to the broadcast characteristic of the TWDM-PON, wavelength assignment provided by the INLP and proposed GA, and the SDN technique.

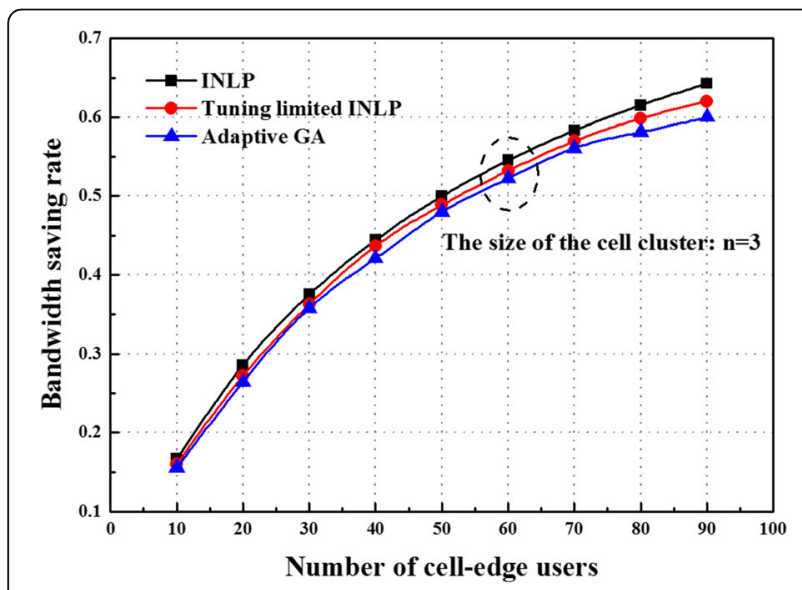

Fig. 8 Bandwidth saving rate considering the number of cell edge users with different methods $(n=3)$

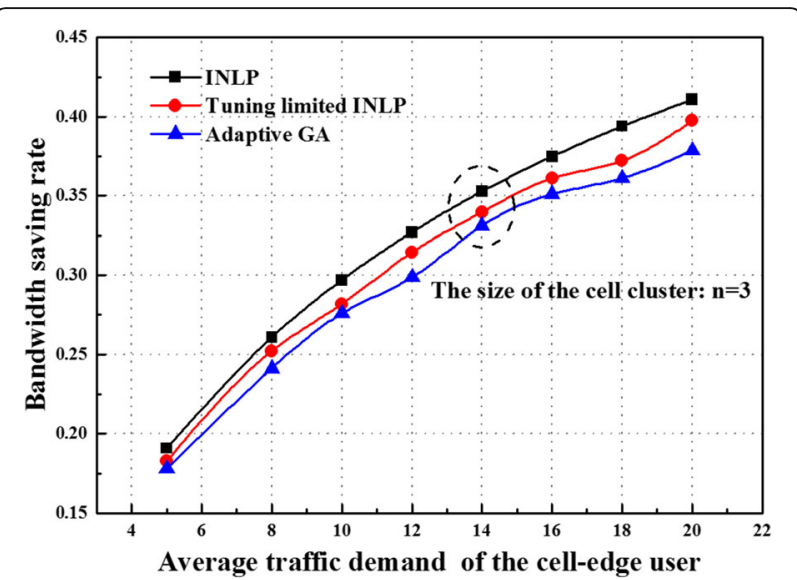

Fig. 9 Bandwidth saving rate considering the average traffic demand of cell edge users with different methods $(n=3)$

\section{Conclusions}

Both INLP model and adaptive GA were explored to release the capacity pressure of the fronthaul, when CoMP technique is introduced in C-RAN. The proposed algorithm offered an efficient way to face the capacity pressure of the fronthaul. Besides, optical resource allocation problem was also considered. The results from the simulations of the proposed algorithm in the 32-cell topology indicated that good performance could be achieved by using the INLP and the proposed adaptive GA. The significant performance was attributed to the broadcast characteristic of the TWDM-PON, wavelength assignment provided by the INLP and the GA, and the SDN technique used in the C-RAN.

\section{Abbreviations}

BBU: Baseband unit; BS: Base station; CoMP: Coordinated multipoint; CPRI: Common public radio interface; C-RAN: Cloud radio access network; D2D: Device-to-device; EE: Energy efficiency; GA: Genetic algorithm; ICI: Intercell interference; INLP: Integer non-linear programming; OFDMA: Orthogonal frequency division multiplexing; OLT: Optical line terminal; ONU: Optical network unit; RRU: Remote radio unit; SE: Spectral efficiency; TWDM-PON: Time wavelength division multiplexing passive optical network; SDN: Software defined network; SE: Spectral efficiency

\section{Acknowledgements}

This work was jointly supported by National High Technology Research and Development Program of China (863 Program) under Grant No. 2015AA015503, the National Natural Science Foundation of China under Grant No. 61372118, and the Beijing Natural Science Foundation under Grant No. 4142036, and Funds of Beijing Advanced Innovation Center for Future Internet Technology of Beijing University of Technology (BJUT), People's Republic of China.

\section{Competing interests}

The authors declare that they have no competing interests.

\section{Author details}

${ }^{1}$ Beijing Key Laboratory of Network System Architecture and Convergence, School of Information and Communication Engineering, Beijing University of Posts and Telecommunications, Beijing 100876, China. ${ }^{2}$ Beijing Advanced Innovation Center for Future Internet Technology, Beijing University of Technology, Beijing 100124, China. ${ }^{3}$ State Key Laboratory of Information Photonics and Optical 
Communications, School of Information and Communication Engineering, Beijing University of Posts and Telecommunications, Beijing 100876, China.

\section{Received: 31 March 2016 Accepted: 15 September 2016} Published online: 21 September 2016

\section{References}

1. V Jungnickel, K Manolakis, W Zirwas, B Panzner, V Braun, M Lossow, R Apelfrojd, The role of small cells, coordinated multipoint, and massive mimo in $5 \mathrm{G}$. IEEE Commun. Mag. 52(5), 44-51 (2014). doi:10.1109/MCOM.2014.6815892

2. N Bhushan, J Li, D Malladi, D Malladi, R Gilmore, D Brenner, A Damnjanovic, R Sukhavasi, C Patel, S Geirhofer, Network densification: the dominant theme for wireless evolution into 5G. IEEE Commun. Mag. 52(2), 82-89 (2014). doi:10.1109/MCOM.2014.6736747

3. BB Haile, AA Dowhuszko, J Hämäläinen, R Wichman, Z Ding, On performance loss of some CoMP techniques under channel power imbalance and limited feedback. IEEE Trans. Wirel. Commun. 14(8), 4469-4481 (2015). doi:10.1109/ TWC.2015.2421898

4. MK Karakayali, GJ Foschini, RA Valenzuela, Network coordination for spectrally efficient communications in cellular systems. IEEE Trans. Wirel. Commun. 13(4), 56-61 (2006). doi:10.1109/MWC.2006.1678166

5. C Yang, S Han, X Hou, AF Molisch, How do we design CoMP to achieve its promised potential? IEEE Trans. Wirel. Commun. 20(1), 67-74 (2013). doi:10. 1109/MWC.2013.6472201

6. WS Soh, HS Kim, A predictive bandwidth reservation scheme using mobile positioning and road topology information. IEEE/ACM Trans. 14(5), 1078-1091 (2006). doi:10.1109/TNET.2006.882899

7. F Song, R Li, H Zhou, Feasibility and issues for establishing network-based carpooling scheme. Pervasive Mob. Comput. 24, 4-15 (2015). doi:10.1016/j. pmcj.2015.05.002

8. H Weingarten, Y Steinberg, S Shamai, The capacity region of the Gaussian multiple-input multiple-output broadcast channel. IEEE Trans. Inf. Theory 52(9), 3936-3964 (2006). doi:10.1109/TIT.2006.880064

9. QH Spencer, AL Swindlehurst, M Haardt, Zero-forcing methods for downlink spatial multiplexing in multiuser MIMO channels. IEEE T. Signal. Proces. 52(2), 461-471 (2004). doi:10.1109/TSP.2003.821107

10. Y Huang, G Zheng, M Bengtsson, KK Wong, L Yang, B Ottersten, Distributed multicell beamforming design approaching Pareto boundary with max-min fairness. IEEE Trans. Wirel. Commun. 11(8), 2921-2933 (2012). doi:10.1109/ TWC.2012.061912.111751

11. B Du, C Pan, W Zhang, M Chen, Distributed energy-efficient power optimization for CoMP systems with max-min fairness. IEEE Commun. Lett. 18(6), 999-1002 (2014). doi:10.1109/LCOMM.2014.2317734

12. Z Xu, C Yang, GY Li, Y Liu, Energy-efficient CoMP precoding in heterogeneous networks. IEEE T. Signal Proces. 62(4), 1005-1017 (2014). doi: 10.1109/TSP.2013.2296279

13. KMS Huq, S Mumtaz, J Bachmatiuk, J Rodriguez, Green HetNet CoMP: energy efficiency analysis and optimization. IEEE T. Veh. Technol. 64(10), 4670-4683 (2015). doi:10.1109/TVT.2014.2371331

14. X Yang, Y Wang, T Zhang, L Cuthbert, L Xiao, Combining CoMP with semismart antennas to improve performance. Electron. Lett. 47(13), 775-776 (2011). doi:10.1049/el.2011.0736

15. Q Cui, H Wang, P Hu, X Tao, P Zhang, J Hamalainen, L Xia, Evolution of limited-feedback CoMP systems from 4G to 5G: CoMP features and limitedfeedback approaches. IEEE Veh. Technol. Mag. 9(3), 94-103 (2014). doi:10. 1109/MVT.2014.2334451

16. $\quad F$ Chen, $W X u, S L i, J R$ Lin, Non-ideal backhaul based spectrum splitting and power allocation for downlink CoMP in cognitive Macro/Femtocell networks. IEEE Commun. Lett. 18(6), 1031-1034 (2014). doi:10.1109/LCOMM.2014.2317774

17. G Nigam, P Minero, M Haenggi, Coordinated multipoint joint transmission in heterogeneous networks. IEEE Trans. Commun. 62(11), 4134-4146 (2014). do: $10.1109 /$ /TCOMM.2014.2363660

18. $\vee \mathrm{Ha}, \mathrm{L} L \mathrm{~L}, \mathrm{ND}$ Dao, Coordinated multipoint (CoMP) transmission design for cloud-RANs with limited fronthaul capacity constraints. IEEE T. Veh. Technol. to be published. doi:10.1109/TVT.2015.2485668

19. China Mobile Research Institute, C-RAN: the road towards green RAN. whitepaper v. 3.0, online, China Mobile, 2013.

20. Y Shi, J Zhang, KB Letaief, Group sparse beamforming for green Cloud-RAN. IEEE T. WIREL. COMMUN. 13(5), 2809-2823 (2013). doi:10.1109/TWC.2014.040214. 131770
21. A Pizzinat, P Chanclou, F Saliou, T Diallo, Things you should know about fronthaul. J. Lightwave Technol 33(5), 1077-1083 (2015). doi:10.1109/JLT. 2014.2382872

22. M Peng, C Wang, V Lau, HV Poor, Fronthaul-constrained cloud radio access networks: insights and challenges. IEEE Wirel. Commun. 22(2), 152-160 (2015). doi:10.1109/MWC.2015.7096298

23. X Liu, F Effenberger, N Chand, L Zhou, H Lin, in 2015 OSA Optical Fiber Communications Conference and Exhibition (OFC). Demonstration of bandwidth-efficient mobile fronthaul enabling seamless aggregation of 36 E-UTRA-like wireless signals in a single 1.1-GHz wavelength channel, (2015), paper.M2J.2. doi:10.1364/OFC.2015.M2J.2

24. M Zhu, X Liu, N Chand, F Effenberger, GK Chang, in 2015 OSA Optical Fiber Communications Conference and Exhibition (OFC). High-capacity mobile fronthaul supporting LTE-advanced carrier aggregation and $8 \times 8 \mathrm{MIMO}$, (2015), paper M2J.3. doi:10.1364/OFC.2015.M2J.3

25. C Liu, J Wang, L Cheng, M Zhu, GK Chang, Key microwave-photonics technologies for next-generation cloud-based radio access networks. J. Lightwave Technol. 32(20), 3452-3460 (2014). doi:10.1109/JLT.2014. 2338854

26. C Neda, T Akihiro, K Konstantinos, W Ting, SDN-controlled topologyreconfigurable optical mobile fronthaul architecture for bidirectional CoMP and low latency inter-cell D2D in the $5 \mathrm{G}$ mobile era. Opt. Express. 22(17), 20809-15 (2014). doi:10.1364/OE.22.020809

27. M Fiorani, A Rostami, L Wosinska, P Monti, Transport abstraction models for an SDN-controlled centralized RAN. IEEE Commun. Lett. 19(8), 1406-1409 (2015). doi:10.1109/LCOMM.2015.2446480

28. B Schrenk, G Humer, M Stierle, H Leopold, Fully-passive remote radio head for uplink cell densification in wireless access networks. IEEE Photon. Technol. Lett. 27(9), 970-973 (2015). doi:10.1109/LPT.2015. 2399231

29. J Li, M Peng, A Cheng, Y Yu, Resource allocation optimization for delaysensitive traffic in fronthaul constrained cloud radio access Networks. IEEE Syst. J. 7335(1), 1-12 (2014). doi:10.1109/JSYST.2014.2364252

30. F Song, Y Zhang, Z An, H Zhou, I You, The correlation study for parameters in four tuples. Int. J. Ad Hoc Ubig. Co. 19(1/2), 38-49 (2015). doi: 10.1504/ IJAHUC.2015.069492

31. A Macho, M Morant, R Llorente, Next-generation optical fronthaul systems using multicore fiber media. J. Lightwave Technol. to be published. doi:10. 1109/JLT.2016.2573038

32. Y Ji, X Wang, S Zhang, R Gu, T Guo, Z Ge, Dual-layer efficiency enhancement for future passive optical network. Sci. China Inf. Sci. 59(2), 1-13 (2016). doi:10. 1007/s11432-015-5430-7

33. YJ Liu, L Guo, LC Zhang, JZ Yang, A new integrated energy-saving scheme in green Fiber-Wireless (FiWi) access network. Sci. China Inf. Sci. 57(6), 1-15 (2014). doi: 10.1007/s11432-013-4958-7

34. I Daisuke, K Shigeru, K Jun-Ichi, T Jun, Dynamic TWDM-PON for mobile radio access networks. Opt. Express 21(22), 26209-26218 (2013). doi:10.1364/OE. 21.026209

35. HX Wang, JX Zhao, H Li, YF Ji, Opaque virtual network mapping algorithms based on available spectrum adjacency for elastic optical networks. Sci. China Inform. Sci, 59(4), 1-11 (2016). doi: 10.1007/s11432-016-5525-9

36. F Song, D Huang, H Zhou, I You, An optimization-based scheme for efficient virtual machine placement. Int. J. Parallel Prog. 42(5), 1-20 (2014). doi:10.1007/s10766-013-0274-5

37. S Nanba, A Agata, in 2013 IEEE $24^{\text {th }}$ International Symposium on Personal Indoor and Mobile Radio Communications (PIMRC). A new IQ data compression scheme for front-haul link in Centralized RAN, (2013), pp. 210-214. doi:10.1109/PIMRCW.2013.6707866

38. KF Nieman, BL Evans, in 2013 IEEE Global Conference on Signal and Information Processing (GlobalSIP). Time-domain compression of complexbaseband LTE signals for cloud radio access networks, (2013), pp. 11981201. doi:10.1109/GlobalSIP.2013.6737122

39. B Guo, W Cap, A Tao, D Samardzija, LTE/LTE-A signal compression on the CPRI Interface. Bell Labs Tech. J. 18(2), 117-133 (2013). doi:10.1002/bltj. 21608

40. D Whitley, A genetic algorithm tutorial. Stat. Comput. 4(2), 65-85 (1994). doi:10.1007/BF00175354

41. J. Koza, Genetic programming: on the programming of computers by means of natural selection, Cambridge, Mass.: MIT, 1992

42. BL Miller, DE Goldberg, Genetic algorithms, tournament selection, and the effects of the noise. Stat. Comput. 9(3), 193-212 (1995) 
43. M Srinivas, LM Patnaik, Adaptive probabilities of crossover and mutation in genetic algorithms. IEEE Trans. Syst. Man Cybern 24(4), 656-667 (1994). doi: $10.1109 / 21.286385$

44. L Gong, X Zhou, W Lu, Z Zhu, A two-population based evolutionary approach for optimizing routing, modulation and spectrum assignments (RMSA) in O-OFDM networks. IEEE Commun. Lett. 16(9), 1520-1523 (2012). doi:10.1109/LCOMM.2012.070512.120740

\section{Submit your manuscript to a SpringerOpen ${ }^{\circ}$ journal and benefit from:}

- Convenient online submission

- Rigorous peer review

- Immediate publication on acceptance

- Open access: articles freely available online

- High visibility within the field

Retaining the copyright to your article

Submit your next manuscript at $>$ springeropen.com 